\title{
Predicting Post-Transplant Recurrence of IgA Nephropathy: The Importance of Crescents
}

\author{
Rupali S. Avasare ${ }^{a}$ Paul E. Rosenstiel ${ }^{b}$ Ziad S. Zaky ${ }^{d}$ Demetra S. Tsapepas ${ }^{c}$ \\ Gerald B. Appel $^{\mathrm{a}}$ Glen S. Markowitz ${ }^{\mathrm{b}}$ Andrew S. Bomback ${ }^{\mathrm{a}}$ Pietro A. Canetta ${ }^{\mathrm{a}}$ \\ ${ }^{a}$ Division of Nephrology, Department of Medicine, and b Division of Renal Pathology, Department of Pathology \\ and Cell Biology, Columbia University College of Physicians and Surgeons, and ' Department of Pharmacy, \\ New York-Presbyterian Hospital, New York, N.Y., and d Division of Nephrology, Glickman Urology and Nephrology \\ Institute, Cleveland Clinic, Cleveland, Ohio, USA
}

\section{Key Words}

Crescentic glomerulonephritis - Glomerulonephritis - Graft function · IgA nephropathy · Kidney transplantation

\begin{abstract}
Background: Most studies that have assessed the predictors of recurrent IgA nephropathy (IgAN) in the renal allograft have focused on post-transplant features. Identifying highrisk pre-transplant features of IgAN is useful for counseling patients and may help in tailoring post-transplant immunosuppression. Methods: We investigated the pre-transplant clinical and biopsy features of 62 patients with IgAN who received transplants at Columbia University Medical Center from 2001 to 2012 and compared the characteristics and outcomes of patients with IgAN recurrence to those without recurrence. The primary outcome was time to recurrent IgAN. Secondary outcomes were a composite of doubling of creatinine or allograft failure, and recurrent IgAN as a cause of allograft dysfunction. Results: Of the 62 patients, 14 had recurrent IgAN in the allograft. Mean time to recurrence was 2.75 years. Those with recurrent disease were younger at the time of native kidney biopsy ( 29 vs. 41 years, $p<0.0009$ ). Black race and Hispanic ethnicity composed a higher propor-
\end{abstract}

\section{KARGER}

(c) 2017 S. Karger AG, Basel

E-Mail karger@karger.com

www.karger.com/ajn tion of the recurrent disease group. On multivariable analysis, significant predictors of recurrent IgAN included age at diagnosis (hazards ratio (HR) $0.911,95 \% \mathrm{Cl} 0.85-0.98$ ), burden of crescents on native biopsy (HR 1.21 per $10 \%$ increase in crescents, $95 \% \mathrm{Cl} 1.00-1.47)$ and allograft rejection (HR $3.59,95 \% \mathrm{Cl} 1.10-11.7)$. Conclusions: Features of native IgAN can help predict the risk of recurrent disease in the renal allograft. In particular, immunologically active disease represented by earlier age of onset and greater burden of crescents on native biopsy is more likely to recur after transplant.

(c) 2017 S. Karger AG, Basel

\section{Introduction}

$\operatorname{Ig} \mathrm{A}$ nephropathy (IgAN) is a major contributor to the worldwide burden of end-stage renal disease (ESRD) [1]. Approximately $30-50 \%$ of patients with IgAN will progress to ESRD over 30 years $[2,3]$. Compared to the general ESRD population, IgAN patients are substantially more likely to be transplanted, receive transplants at a younger age and have lower post-transplant mortality [46]. However, long-term allograft survival is no better for IgAN patients compared to those with other causes of 
ESRD [7], and this is at least partly due to disease recurrence.

IgAN may recur in the renal allograft in $9-61 \%$ of patients [8]. Recurrent disease was thought to have little impact on graft outcomes [9]. However, recent retrospective studies with longer duration of follow-up suggest that recurrent disease contributes substantially to allograft damage $[6,10,11]$. Risk factors for recurrence include presence of specific HLA genotypes, zero mismatch kidneys, high serum IgA levels, choice of induction therapy, choice of maintenance immunosuppression and duration of follow-up after transplant [12-16]. Post-transplant crescentic IgAN confers poorer allograft outcomes compared to post-transplant recurrence of non-crescentic IgAN [17, 18]. Most studies assessing the predictors of recurrent IgAN have focused on post-transplant clinical features. Improving clinicians' ability to estimate risk of recurrence prior to the transplant would be useful, particularly for counseling patients and designing management strategies.

Here we investigated the pre-transplant clinical and biopsy features of patients with IgAN who received renal transplants at Columbia University Medical Center (CUMC) from 2001 to 2012 and compare the characteristics and graft outcomes of patients with IgAN recurrence to those without recurrent disease.

\section{Methods}

\section{Subjects}

During the period from January 2001 through December 2012, 2,256 adult ( $\geq 18$ years) patients with unique medical record numbers underwent renal transplantation at CUMC, including $150 \mathrm{pa}$ tients with ESRD resulting from biopsy-documented IgAN. Only patients whose native kidney biopsies were read or reviewed at CUMC were included in this study. Secondary IgAN and SLE were excluded by history and clinical features. One patient had a history of Henoch-Schonlein purpura and IgAN on biopsy. Clinical and pathology data for 62 of the 150 patients was retrospectively reviewed. Patients were stratified into 2 groups, IgAN recurrence and no-IgAN recurrence for comparison of risk factors.

\section{Histopathological Evaluation}

All native biopsies were processed by standard techniques for light microscopy, immunofluorescence (IF) and electron microscopy (EM). Each biopsy was independently re-reviewed and scored by a single pathologist (P.E.R.). The diagnosis of IgAN was based on the presence of dominant or codominant staining for IgA by IF, with confirmatory EM findings of electron dense deposits in the majority of cases. Each biopsy was graded according to the Oxford classification ('MEST'), including scores for mesangial hypercellularity (M), endocapillary hypercellularity (E), segmental glomerulosclerosis (S) and tubulointerstitial fibrosis (T) [19]. Crescents were categorized as cellular, fibrocellular or fibrous. Burden of crescents was calculated for each biopsy as the percentage of nonsclerotic glomeruli with cellular or fibrocellular crescents among all open glomeruli. Purely fibrous crescents were excluded from the numerator due to the lack of consensus in identifying fibrous crescents, and globally sclerotic glomeruli were excluded from the denominator.

\section{Outcomes and Definitions}

The primary outcome was time to recurrence of IgAN. The 2 secondary outcomes were (1) composite of doubling of serum creatinine (compared to post-transplant nadir) or allograft failure and (2) recurrent IgAN as a cause of allograft dysfunction (determined by the treating clinician with concurrence by the pathologist). We chose recurrence as the primary outcome because this can be diagnosed on biopsy and analyzed in an objective time-toevent fashion.

Pre-transplant variables that were collected included demographic data (age, gender, ethnicity), clinical presentation at time of biopsy, time from first biopsy to ESRD (defined as initiation of dialysis or transplantation) and duration of dialysis. Annualized loss of eGFR may have been a more meaningful measure of pretransplant disease activity [20]; however, creatinine at the time of biopsy was not consistently documented. Post-transplantation data included allograft type (living vs. deceased donor), induction and maintenance immunosuppression, biopsy proven allograft rejection and grade, BK viremia and/or nephropathy, occurrence of proteinuria, nephrotic syndrome (defined as proteinuria $>3.5$ g per gram of creatinine, hypoalbuminemia and clinical documentation), hypertension (defined as BP $>140 / 90 \mathrm{~mm} \mathrm{Hg}$ or need for anti-hypertensives to achieve a BP of $<140 / 90 \mathrm{~mm} \mathrm{Hg}$ ), graft loss (defined as return to dialysis or re-transplantation) and mortality.

\section{Statistical Analysis}

Descriptive statistics for continuous variables are presented as the mean \pm SD if parametric, or median and interquartile range if non-parametric. For categorical variables, data are presented as count with percentage. Inter-group comparisons were made by Student's t test for normally distributed continuous variables, the Mann-Whitney $U$ test for nonparametric continuous variables and Fisher's exact test for categorical variables. Univariate and multivariate Cox proportional hazards models were created for the time-dependent outcomes of recurrent IgAN and the composite of allograft failure or doubling creatinine. Logistic regression models were created for the outcome of recurrent IgAN as a cause of allograft dysfunction. All $\mathrm{p}$ values were 2 -tailed and values $<0.05$ were considered statistically significant. Analyses were performed using SAS version 9.4 (SAS Institute Inc., Cary, N.C., USA).

\section{Results}

\section{Clinical Characteristics Pre-Transplant}

We reviewed the charts and original native kidney biopsies of $62 \operatorname{IgAN}$ patients who received a transplant at our center and whose native biopsies had been read at our center. Of these 62 patients, 14 had recurrent IgAN in the allograft. Baseline characteristics of the cohort, in- 
Table 1. Baseline characteristics prior to renal transplantation

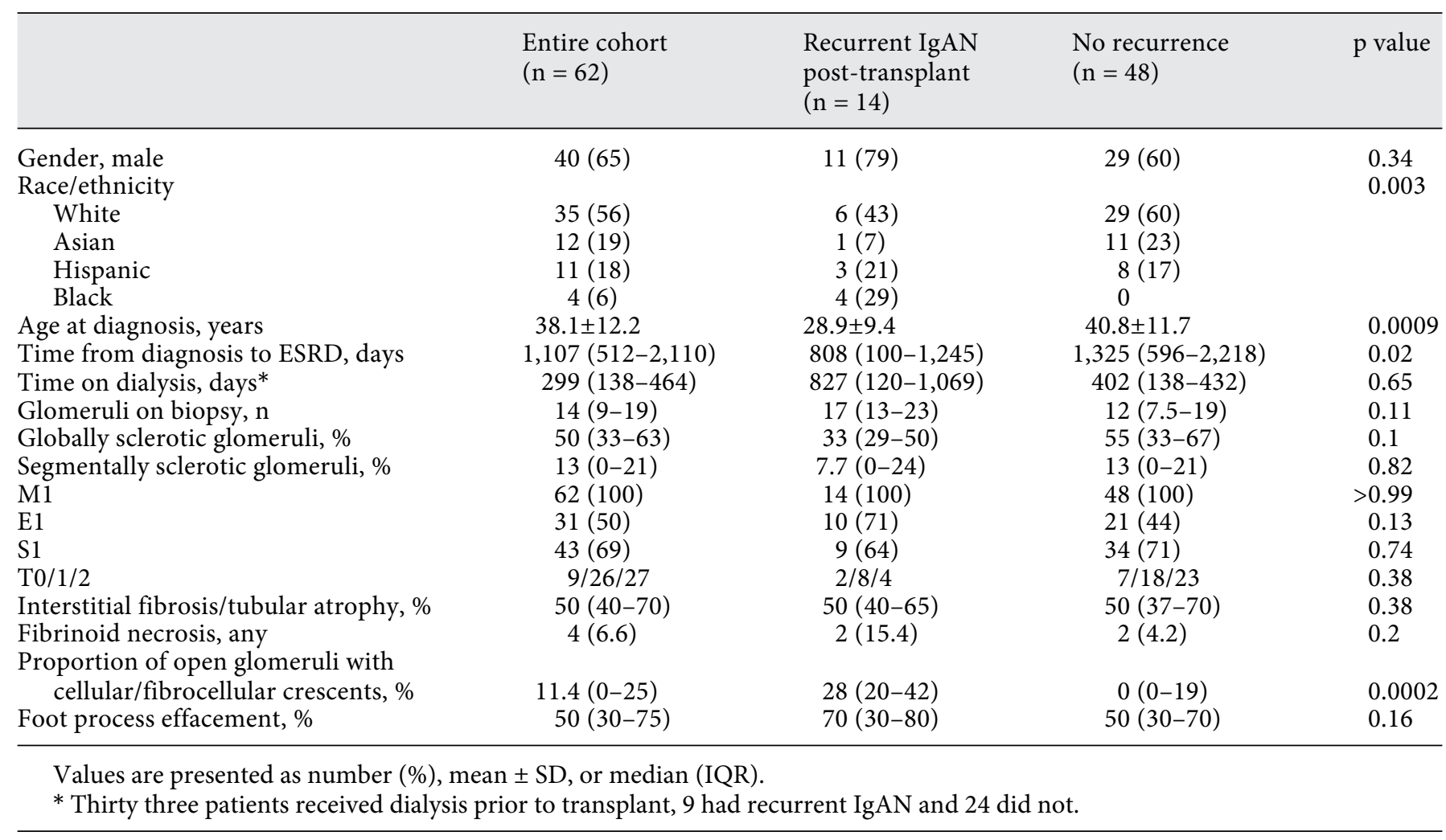

cluding demographics and biopsy features from the native diagnostic biopsy, are presented in table 1. Those who experienced recurrent disease were significantly younger at the time of native kidney biopsy than those who did not have recurrence ( 29 vs. 41 years, $p<0.0009$ ). Black race and Hispanic ethnicity composed a higher proportion of the recurrent disease group than the norecurrence group. Progression from native kidney biopsy to ESRD was significantly more rapid in those who developed post-transplant IgAN recurrence versus in those who did not have recurrence (median 808 vs. 1,325 days, $\mathrm{p}=0.02$ ).

\section{Histopathological Characteristics}

There were no differences in MEST classification, percent interstitial fibrosis and tubular atrophy, foot process effacement on native kidney biopsy in those who had post-transplant IgAN recurrence versus in those with no recurrence (table 1). All 62 patients were positive for the Oxford 'M' classifier of mesangial hypercellularity. Of the 62 native biopsies, $47 \%$ had $<10 \%$ crescents, $29 \%$ had 10 $29 \%$ crescents, $11 \%$ had $30-49 \%$ crescents and $13 \%$ had $\geq 50 \%$ crescents. Of those who had recurrent IgAN, the

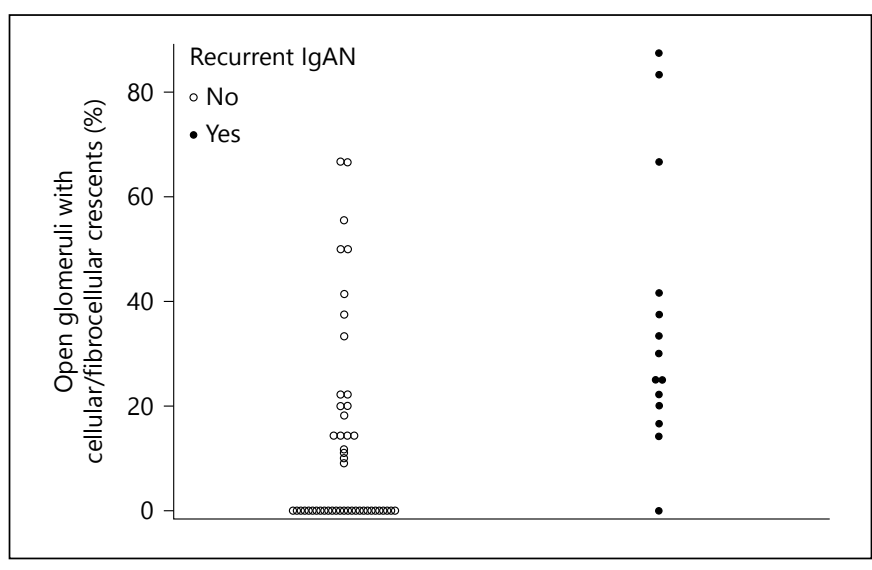

Fig. 1. Percentage of open glomeruli with cellular or fibrocellular crescents among patients with post-transplant recurrent IgAN (closed circles) and those without recurrent IgAN (open circles).

median percentage of open glomeruli containing cellular or fibrocellular crescents on native biopsy was $28 \%$ compared to $0 \%$ in those without recurrent disease $(\mathrm{p}=$ $0.0002)$. The distribution of crescents is shown in figure 1. Among those with recurrent IgAN, 13 of 14 (93\%) pa- 
Table 2. Transplant and post-transplant characteristics

\begin{tabular}{|c|c|c|c|c|}
\hline & $\begin{array}{l}\text { Entire cohort } \\
(n=62)\end{array}$ & $\begin{array}{l}\text { Recurrent IgAN } \\
\text { post-transplant }(\mathrm{n}=14)\end{array}$ & $\begin{array}{l}\text { No recurrence } \\
(\mathrm{n}=48)\end{array}$ & $\mathrm{p}$ value \\
\hline Age at transplant, years & $42.7 \pm 13.0$ & $32.5 \pm 9.9$ & $45.7 \pm 12.4$ & 0.0006 \\
\hline Preemptive, \% & $29(47)$ & $5(36)$ & $24(50)$ & 0.38 \\
\hline Thymoglobulin induction & $49(86)$ & $8(73)$ & $41(89)$ & 0.17 \\
\hline Standard maintenance therapy* & $48(77)$ & $10(71)$ & $38(79)$ & 0.72 \\
\hline ACEI and/or ARB use & $26(42)$ & $8(57)$ & $18(38)$ & 0.23 \\
\hline Hypertension & $41(66)$ & $9(64)$ & $32(67)$ & $>0.99$ \\
\hline Diabetes mellitus & $12(29)$ & $2(14)$ & $10(21)$ & 0.72 \\
\hline Any allograft biopsy & $47(76)$ & $14(100)$ & $33(69)$ & 0.01 \\
\hline Rejection, any & $17(27)$ & $6(43)$ & $11(23)$ & 0.18 \\
\hline Rejection, cellular & $15(24)$ & $6(43)$ & $9(19)$ & 0.08 \\
\hline Serum creatinine at IgAN recurrence, $\mathrm{mg} / \mathrm{dl}$ & & $2.20(1.75-2.74)$ & & NA \\
\hline Proteinuria at IgAN recurrence, $\mathrm{mg} / \mathrm{gCr}$ & & $590(319-977)$ & & NA \\
\hline Microhematuria at recurrence & & $7(78)$ & & NA \\
\hline Serum creatinine at last follow-up, $\mathrm{mg} / \mathrm{dl}(\mathrm{n}=51)$ & $1.30(1.09-1.65)$ & $2.01(1.55-2.69)$ & $1.21(1.01-1.55)$ & 0.001 \\
\hline Proteinuria at last follow-up, mg/gCr $(\mathrm{n}=40)$ & $114(100-367)$ & $541(187-871)$ & $100(100-252)$ & 0.06 \\
\hline Microhematuria at last follow-up $(\mathrm{n}=31)$ & $8(26)$ & $3(60)$ & $5(19)$ & 0.09 \\
\hline Allograft failure & $11(18)$ & $6(43)$ & $5(10)$ & 0.01 \\
\hline Doubled serum creatinine & $11(28)$ & $7(50)$ & $4(8.3)$ & 0.001 \\
\hline Composite outcome & $12(29)$ & $7(50)$ & $5(10)$ & 0.003 \\
\hline Time from transplant to event or censoring, days & $1,763(1,154-2,961)$ & $1,487(1,112-2,566)$ & $1,901(1,209-2,978)$ & 0.38 \\
\hline
\end{tabular}

Values are presented as number (\%), mean $\pm \mathrm{SD}$, or median (IQR).

* Defined as calcineurin inhibitor plus mycophenolate mofetil or mycophenolic acid.

tients had at least $10 \%$ crescents on their native biopsies, and among those who did not recur only 20 of 48 patients (42\%) had at least $10 \%$ crescents on their native biopsies $(\mathrm{p}=0.007)$.

\section{Clinical Characteristics Post-Transplant}

Characteristics of the kidney transplants and posttransplant course are summarized in table 2. Age at transplant was significantly lower in those who had recurrent IgAN compared to those who had no recurrence (mean 32.5 vs. 45.7 years, $\mathrm{p}<0.01$ ). Exposure to immunosuppression was similar between the groups. Rejection (either cellular or humoral), calcineurin toxicity (as documented in notes or pathology) and BK viremia was no different between the 2 groups. Nadir creatinine (1.6 vs. $1.17, \mathrm{p}=0.005)$ and creatinine at last follow-up (2.01 vs. $1.21, \mathrm{p}=0.001)$ were significantly higher in those with recurrent IgAN.

\section{Recurrence and Treatment}

Mean time to diagnosis of recurrent IgAN on allograft biopsy was 2.75 years after transplant (median 1.25 years). At the time of recurrence, the median proteinuria was 590 $\mathrm{mg} / \mathrm{g}$ creatinine and the median serum creatinine was $2.2 \mathrm{~g} / \mathrm{dl}$. Of the 14 recurrences, 9 patients did not receive specific therapy beyond standard transplant immunosuppression, 2 patients received high-dose corticosteroid therapy alone, 2 patients received corticosteroid therapy and then rituximab therapy for a second recurrence and 1 patient received corticosteroid therapy plus rituximab therapy for a second recurrence plus ACTH for a third recurrence.

\section{Primary Outcome}

Univariate hazards ratios (HRs) for the primary outcome of time to recurrent IgAN after transplant are presented in table 3. By univariate analyses, significant pre- 
Table 3. Univariable cox proportional hazards for time from transplant to diagnosis of recurrent IgAN

\begin{tabular}{|c|c|c|c|}
\hline Variable & HR & $95 \% \mathrm{CI}$ & $\mathrm{p}$ value \\
\hline \multicolumn{4}{|l|}{ Baseline characteristics } \\
\hline Age at diagnosis (per year) & 0.91 & $0.85-0.97$ & 0.003 \\
\hline Gender, male & 2.56 & $0.70-9.35$ & 0.15 \\
\hline Black race (vs. other) & 7.14 & $2.09-24.4$ & 0.002 \\
\hline Time from diagnosis to ESRD (per year) & 0.79 & $0.61-1.03$ & 0.09 \\
\hline \multicolumn{4}{|l|}{ Native biopsy characteristics } \\
\hline \multicolumn{4}{|c|}{ Proportion of open glomeruli with cellular/fibrocellular } \\
\hline E1 & 2.61 & $0.81-8.35$ & 0.11 \\
\hline S1 & 0.72 & $0.24-2.16$ & 0.56 \\
\hline T1 (vs. T0) & 1.18 & $0.25-5.62$ & 0.84 \\
\hline T2 (vs. T0) & 0.49 & $0.085-2.77$ & 0.42 \\
\hline IgG deposition (vs. none) & 0.91 & $0.24-3.43$ & 0.89 \\
\hline \multicolumn{4}{|l|}{ Transplant characteristics } \\
\hline Preemptive transplant & 0.55 & $0.18-1.65$ & 0.29 \\
\hline Living donor & 1.75 & $0.38-8.00$ & 0.47 \\
\hline Rejection, any & 3.26 & $1.05-10.2$ & 0.04 \\
\hline Thymoglobulin induction (vs. other) & 0.58 & $0.12-2.75$ & 0.49 \\
\hline Standard maintenance therapy (vs. other) & 0.94 & $0.28-3.16$ & 0.92 \\
\hline Corticosteroid use & 2.03 & $0.62-6.63$ & 0.24 \\
\hline ACEI and/or ARB use post-transplant & 1.95 & $0.68-5.63$ & 0.22 \\
\hline
\end{tabular}

dictors of recurrent IgAN in the allograft included age at diagnosis, black race, burden of crescents on native biopsy and a diagnosis of allograft rejection. There was a suggestion that a longer time from diagnosis to ESRD was associated with a lower hazard of recurrent $\operatorname{IgAN}$, but this did not reach statistical significance (HR 0.79, 95\% CI 0.61-1.03, per year). In sensitivity analyses, a greater amount of crescents remained significantly associated with shorter time to recurrence when examined by tertile or as a binary variable. Figure 2a shows Kaplan-Meier curves for time to recurrent IgAN for those with $<10$ versus $\geq 10 \%$ crescents on native biopsy.

On multivariate analysis, significant predictors of recurrent IgAN included age at diagnosis (HR 0.911, 95\% CI 0.85-0.98), burden of crescents on native biopsy (HR 1.21 per $10 \%$ increase in crescents, $95 \%$ CI $1.00-1.47$ ) and allograft rejection (HR 3.59, 95\% CI 1.10-11.7), after adjusting for sex, ethnicity, time to ESRD, Oxford 'E' lesion on native biopsy, steroid use and angiotensin-convertingenzyme inhibitor (ACEI)/angiotensin receptor blocker (ARB) use post-transplant.

\section{Secondary Outcomes}

For the composite secondary outcome of doubling of serum creatinine after transplant or allograft failure, significant predictors in univariate Cox analyses included allograft rejection (HR 6.50, 95\% CI 1.97-21.4), recurrent IgAN (HR 4.94, 95\% CI 1.56-15.6) and nadir serum creatinine (HR 2.49 per each $0.5 \mathrm{~g} / \mathrm{dl}$ increase, 95\% CI $1.48-$ 4.18). On multivariate analysis, the only significant predictors of the composite outcome were allograft rejection (HR 4.76, 95\% CI 1.40-16.1) and nadir serum creatinine (HR 2.36 per each $0.5 \mathrm{~g} / \mathrm{dl}$ increase, 95\% CI 1.29-4.33), whereas recurrent IgAN was no longer a significant predictor. Burden of crescents did not significantly predict the composite outcome (univariate $\mathrm{p}=0.36$ ). Figure $2 \mathrm{~b}$ shows Kaplan-Meier curves for time to the composite outcome are shown for the group with $<10$ versus $\geq 10 \%$ crescents on native biopsy.

For the secondary outcome of recurrent IgAN as a cause of allograft dysfunction, univariate logistic regression showed significant associations with age at diagnosis (OR 0.89 per year, 95\% CI 0.82-0.97), burden of crescents on native biopsy (OR 1.52 per $10 \%$ increase in crescents, 95\% CI 1.13-2.02) and the presence of the Oxford ' $E$ ' lesion on native biopsy (OR 12.3, 95\% CI 1.45-104). There was a suggestion of a protective effect with the use of rabbit anti-thymocyte globulin induction, albeit not reaching statistical significance (OR 0.19, 95\% CI 0.03-1.04, $\mathrm{p}=0.06)$. Variables that were not significantly associated with recurrent IgAN as a cause of allograft dysfunction included sex, race/ethnicity, preemptive transplant, liv- 


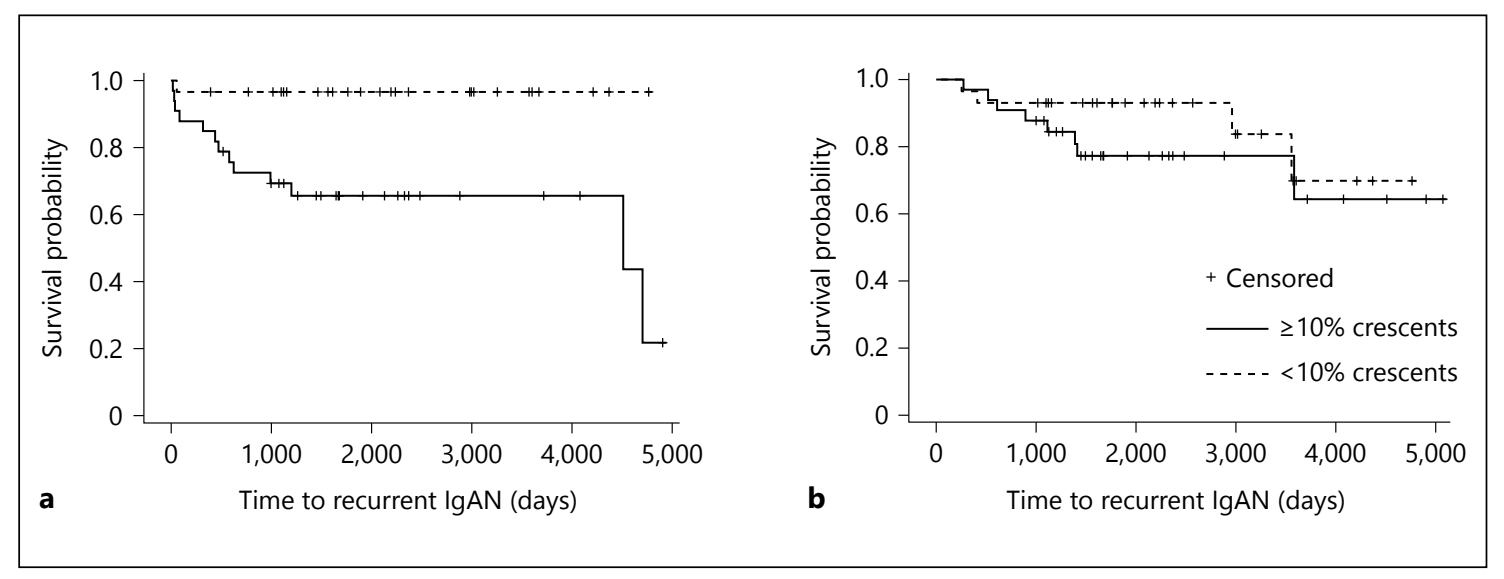

Fig. 2. Kaplan-Meier curves are shown comparing subjects with $<10 \mathrm{vs.} \geq 10 \%$ crescents on native biopsy, for the primary outcome of time to recurrent IgAN post-transplant (a) and the secondary composite outcome of doubling creatinine post-transplant or allograft failure $(\mathbf{b})$.

ing donor transplant, any rejection, type of maintenance immunosuppression, use of corticosteroids or posttransplant ACEI or ARB use. In a multivariate model, age at diagnosis (OR 0.89 per year, 95\% CI 0.81-0.98) and burden of glomeruli on native biopsy (OR 1.55, 95\% CI 1.07-2.25) remained significantly associated with the risk of recurrent IgAN causing allograft dysfunction, after adjusting for age, sex and ethnicity.

\section{Discussion}

We retrospectively analyzed a cohort of patients with IgAN who had native biopsies reviewed and underwent transplantation at CUMC. We compared the pre-transplant clinical and biopsy features of patients with recurrent IgAN with those who had no recurrence. We found that patients who developed recurrent IgAN were younger and had a more rapid progression to ESRD from their native disease. On multivariable analysis, predictors of recurrent IgAN were younger age, burden of crescents in the native kidney biopsy and allograft rejection. Recurrent IgAN was itself a predictor of doubling the serum creatinine and graft failure.

Though the rate of recurrence of IgAN is high (9-61\% in the literature; $22.5 \%$ in this study), the rate of graft loss due to IgAN is thought to be low $(3-4 \%)[8,21]$. Moroni et al. [11] analyzed the post-transplant outcomes of 190 patients with IgAN transplanted between 1981 and 2010. The rate of IgAN recurrence was $37.2 \%$. Of these patients, about half experienced allograft loss and about half of the graft losses were due to recurrent IgAN. In univariate analysis, predictors of IgAN recurrence included year of transplant, younger age of recipient, maintenance immunosuppression without mycophenolate and use of $<3 \mathrm{im}$ munosuppressive agents. IgAN was an independent predictor of worse graft outcomes compared to non-diabetic controls. Choy et al. [10] examined the post-transplant outcomes of 75 patients with IgAN transplanted between 1984 and 2001 and found recurrent disease in 19\% of patients. Of these patients, roughly a third of the patients had graft failure of which $60 \%$ were due to IgAN. Patients with IgAN had superior outcomes compared to controls without IgAN up until 12 years post-transplant when patients with IgAN had poorer graft outcomes. Similar to our study, Choy et al. [10] did not find donor type (living vs. deceased) and immunosuppression to be significant predictors of recurrence.

Clayton et al. [14] found recurrent IgAN as the cause of graft loss in $12.6 \%$ of patients transplanted for IgAN. Interestingly, they also found that patients who were corticosteroid-free at one year were more likely to have graft loss from IgAN than patients who received corticosteroids. Our study did not find that long-term ( $>1$ year) use of corticosteroids reduced the risk of recurrence. We found a suggestion that anti-thymocyte globulin induction was associated with a longer time to recurrence compared to other types of induction (mostly basiliximab in this cohort). While this HR did not reach statistical significance, the effect size was clinically important, and in line with the findings of Berthoux et al. [22] who also found a protective effect with anti-thymocyte globulin.

Our study found that a greater burden of crescents on the native biopsy was associated with a shorter time to 
recurrence of IgAN post-transplant, and was also associated with a greater likelihood that the recurrent IgAN was pathogenic. We did not find that the burden of native crescents affected the composite outcome of doubling serum creatinine or allograft failure, which appeared to be largely driven by nadir creatinine (likely a proxy for allograft quality) and subsequent rejection. Our findings support the hypothesis that crescentic IgAN represents a more severe and aggressive form of disease, and thus, the pathophysiologic process leading to severe disease in the native kidney may lead to more aggressive recurrent disease in the transplanted kidney. In native IgAN, higher grades of proteinuria, hypertension and impaired kidney function are predictors of poor clinical outcomes [23]. However, we did not find these variables to be of significant value in predicting post-transplant recurrence. Thus, we hypothesize that crescents may be more indicative of immunologic disease activity, and thus recurrence risk, while these other factors may be confounded by disease chronicity.

Clinically, patients with crescentic IgAN have a higher incidence of hypertension and nephrotic syndrome [24, 25]. In IgAN, crescents are associated with decreased renal survival, with an inverse correlation between the number of crescents and duration of renal survival [26, 27]. Lv et al. [28] found that in crescentic IgAN, defined as $>50 \%$ crescents, progression to ESRD was as high as $70 \%$ at 5 years. Though the prognostic value of crescents was not systematically assessed in the Oxford cohort [29] due to low prevalence, other retrospective studies that have sought to investigate the prognostic value of crescents when added to the MEST score show conflicting results [27, 30, 31]. Moreover, the percent of crescents that define a biopsy as crescentic IgAN has been variably defined, ranging from any crescents to $50 \%$ glomerular involvement [27, 30, 32]. The largest systematic review and meta-analysis to examine the Oxford classification found that the presence of any crescent had independent prognostic importance, and the authors proposed adding a 'C' lesion (for any crescent) to the classification [33]. Our study supports the association of crescents with adverse outcomes, and further suggests a dose effect where a greater percentage of crescentic glomeruli are associated with higher risks of pathogenic recurrence.

There are several limitations of our study besides its retrospective design and single center design. The sample size is small with only 62 patients as we designed the study to include only those who had their native biopsies reviewed at Columbia. A larger study including multiple centers would have sacrificed the ability to examine his- tology and other variables in detail. Because protocol biopsies were not done routinely, subclinical recurrent IgAN cannot be excluded in the $31 \%$ of patients with 'no recurrence' who had not undergone renal allograft biopsy. Similarly, all subjects with recurrent IgAN had a forcause biopsy for allogfraft dysfunction, and thus, this group was likely enriched with rejection events. Our finding of an association between allograft rejection and time to recurrent IgAN was likely due to a confounding association. We found that Hispanic ethnicity and black race were more prevalent in the recurrent IgAN group, and that black race predicted recurrent IgAN on univariate analysis, but this was based on small numbers (only 4 black patients in the study, all of whom recurred.) Other studies have produced conflicting data on whether black race portends worse prognosis in IgAN $[34,35]$. A strength of the study is that all patients received transplants after 2001 which makes for a more standardized induction and maintenance immunosuppression regimen compared to previous studies that included patients from prior eras.

Our study adds to the growing literature examining the impact of recurrent IgAN on long-term allograft outcomes. Importantly, we have found that younger age at diagnosis and crescentic disease in the native kidney are strongly associated with earlier post-transplant IgAN recurrence. This may be helpful in counseling IgAN patients on their expectations prior to transplant, on selecting post-transplant immunosuppressive regimens and/or in designing strategies for managing recurrent disease. In addition to histologic and clinical predictors of recurrence, future prospective studies may include measurements of novel biomarkers of disease activity to predict recurrent IgAN [36]. Continuing to hone our predictive ability is important, given our findings and those of others that suggest that recurrent IgAN is associated with poorer allograft outcomes.

\section{Disclosure Statement}

The results presented in this paper have not been published previously and are not being considered for publication elsewhere. The authors have no conflicts of interest to disclose.

\section{Financial Disclosures}

Dr. A.S. Bomback is supported by National Institutes of HealthNational Institute on Minority Health and Health Disparities grant R01-MD009223. 


\section{References}

1 Schena FP: A retrospective analysis of the natural history of primary IgA nephropathy worldwide. Am J Med 1990;89:209-215.

-2 D'Amico G: Natural history of idiopathic IgA $>14$ nephropathy: role of clinical and histological prognostic factors. Am J Kidney Dis 2000;36: 227-237.

-3 Moriyama T, et al: Prognosis in IgA nephrop- 15 athy: 30-year analysis of 1,012 patients at a single center in Japan. PLoS One 2014; 9:e91756.

-4 O'Shaughnessy MM, Montez-Rath ME, Lafayette RA, Winkelmayer WC: Patient characteristics and outcomes by GN subtype in ESRD. Clin J Am Soc Nephrol 2015;10: 1170-1178.

5 Saran R, et al: US renal data system 2015 annual data report: epidemiology of kidney disease in the United States. Am J Kidney Dis 2016;67(3 suppl 1):Svii, S1-S305.

6 Wyld ML, Chadban SJ: Recurrent IgA nephropathy after kidney transplantation. Transplantation 2016;100:1827-1832.

7 Andresdottir MB, Haasnoot GW, Doxiadis II, Persijn GG, Claas FH: Exclusive characteristics of graft survival and risk factors in recipients with immunoglobulin A nephropathy: a retrospective analysis of registry data. Transplantation 2005;80:1012-1018.

8 Ponticelli C, Glassock RJ: Posttransplant recurrence of primary glomerulonephritis. Clin J Am Soc Nephrol 2010;5:2363-2372.

$\$ 9$ Berger J: Recurrence of IgA nephropathy in renal allografts. Am J Kidney Dis 1988;12: 371-372.

10 Choy BY, Chan TM, Lo SK, Lo WK, Lai KN Renal transplantation in patients with primary immunoglobulin A nephropathy. Nephro Dial Transplant 2003;18:2399-2404.

11 Moroni G, et al: The long-term outcome of renal transplantation of IgA nephropathy and the impact of recurrence on graft survival. Nephrol Dial Transplant 2013;28:1305-1314.

-12 Andresdottir MB, Haasnoot GW, Persijn GG, Claas FH: HLA-B8, DR3: a new risk factor for graft failure after renal transplantation in patients with underlying immunoglobulin A nephropathy. Clin Transplant 2009;23:660-665.
13 Wang AY, et al: Recurrent IgA nephropathy in renal transplant allografts. Am J Kidney Dis 2001;38:588-596.

14 Clayton P, McDonald S, Chadban S: Steroids and recurrent IgA nephropathy after kidney transplantation. Am J Transplant 2011;11: 1645-1649.

5 Odum J, et al: Recurrent mesangial IgA nephritis following renal transplantation. Nephrol Dial Transplant 1994;9:309-312.

16 Bachman U, et al: The clinical course of IgAnephropathy and Henoch-Schönlein purpura following renal transplantation. Transplantation 1986;42:511-515.

17 Jeong HJ, et alGlomerular crescents are responsible for chronic graft dysfunction in post-transplant IgA nephropathy. Pathol Int 2004;54:837-842.

18 Kowalewska J, et al: IgA nephropathy with crescents in kidney transplant recipients. Am J Kidney Dis 2005;45:167-175.

19 Working Group of the International IgA Nephropathy Network and the Renal Pathology Society, Cattran DC, et al: The Oxford classification of IgA nephropathy: rationale, clinicopathological correlations, and classification. Kidney Int 2009;76:534-545.

20 Bjorneklett R, et al: Pre-transplant course and risk of kidney transplant failure in IgA nephropathy patients. Clin Transplant 2011 25:E356-E365.

21 Ponticelli C, et al: Kidney transplantation in patients with IgA mesangial glomerulonephritis. Kidney Int 2001;60:1948-1954.

22 Berthoux F, et al: Antithymocyte globulin (ATG) induction therapy and disease recurrence in renal transplant recipients with primary IgA nephropathy. Transplantation 2008;85:1505-1507.

23 D'Amico G: Natural history of idiopathic IgA nephropathy and factors predictive of disease outcome. Semin Nephrol 2004;24: 179-196.

24 Bitencourt-Dias C, Bahiense-Oliveira M, Saldanha LB, Barros RT, Woronik V: Comparative study of IgA nephropathy with and without crescents. Braz J Med Biol Res 2004;37: 1373-1377.
25 Xie J, et al: Predicting progression of IgA nephropathy: new clinical progression risk score. PLoS One 2012;7:e38904.

26 Abe T, et al: Participation of extracapillary lesions (ECL) in progression of IgA nephropathy. Clin Nephrol 1986;25:37-41.

27 Lee MJ, et al: Clinical implication of crescentic lesions in immunoglobulin A nephropathy. Nephrol Dial Transplant 2014;29:356364

28 Lv J, et al: Prediction of outcomes in crescentic IgA nephropathy in a multicenter cohort study. J Am Soc Nephrol 2013;24:2118-2125.

-29 Working Group of the International IgA Nephropathy Network and the Renal Pathology Society, Roberts IS, et al: The Oxford classification of IgA nephropathy: pathology definitions, correlations, and reproducibility. Kidney Int 2009;76:546-556.

-30 Katafuchi R, Ninomiya T, Nagata M, Mitsuiki $\mathrm{K}$, Hirakata H: Validation study of Oxford classification of IgA nephropathy: the significance of extracapillary proliferation. Clin J Am Soc Nephrol 2011;6:2806-2813.

-31 Shi SF, et al: Pathologic predictors of renal outcome and therapeutic efficacy in IgA nephropathy: validation of the Oxford classification. Clin J Am Soc Nephrol 2011;6:21752184.

32 Tumlin JA, Lohavichan V, Hennigar R: Crescentic, proliferative IgA nephropathy: clinical and histological response to methylprednisolone and intravenous cyclophosphamide. Nephrol Dial Transplant 2003;18:1321-1329.

$33 \mathrm{Lv}$ J, et al: Evaluation of the Oxford classification of IgA nephropathy: a systematic review and meta-analysis. Am J Kidney Dis 2013;62: 891-899.

34 Arroyo AH, et al: Predictors of outcome for severe IgA Nephropathy in a multi-ethnic U.S. cohort. Clin Nephrol 2015;84:145-155.

- 35 Hall YN, Fuentes EF, Chertow GM, Olson JL: Race/ethnicity and disease severity in IgA nephropathy. BMC Nephrol 2004;5:10.

36 Berthelot L, et al: Recurrent IgA nephropathy is predicted by altered glycosylated IgA, autoantibodies and soluble CD89 complexes. Kidney Int 2015;88:815-822. 